\title{
2006-1836: AN APPROACH TO INTERNATIONAL COMPETITION
}

\section{Frank Barnes, University of Colorado-Boulder}

Frank Barnes received his B.S. in Electrical Engineering in 1954 from Princeton University and his M.S., Engineer, and Ph.D. degrees from Stanford University in 1955, '56, and '58 respectively. He was a Fulbright Scholar in Baghdad, Iraq in 1958 and joined the University of Colorado in 1959 where he is currently a Distinguished Professor. He has served as Chairman of the Department of Electrical Engineering, Acting Dean of the College of Engineering, and in 1971 as cofounder/Director with Professor George Codding of the Political Science Dept. of the Interdisciplinary Telecommunications Program, ITP.

He has served as Chair of the IEEE Electron Device Society, President of the Electrical Engineering Department Heads Association, Vice President of IEEE for Publications, Editor of the IEEE Student Journal and the IEEE Transactions on Education as well as President of the Bioelectromagnetics Society and U.S. Chair of Commission K-URSI. He is a Fellow of AAAS, IEEE, International Engineering Consortium and he is a member of the National Academy of Engineering.

Dr. Barnes has been awarded the Curtis McGraw Research Award from ASEE, the Leon Montgomery Award from the International Communications Association, the 2003 IEEE Education Society Achievement Award, Distinguished Lecturer for IEEE Electron Device Society, the 2002 ECE Distinguished Educator Award from ASEE, The Colorado Institute of Technology Catalyst Award 2004, and the Bernard M. Gordon Prize from National Academy of Engineering for Innovations in Engineering Education $2004 \mathrm{He}$ was born in Pasadena, California in 1932 and attended numerous elementary schools throughout the country. He and his wife, Gay, have two children and two grandchildren 


\title{
EDUCATING OUR ENGINEERS FOR GLOBAL COMPETITION
}

\begin{abstract}
.
With the rapid growth in the number of engineering graduates in Asia and around the world an important question is: how should we be preparing the undergraduate in the US to compete? Asia is graduating more than half a million engineers a year compared to roughly sixty thousand in the US and starting wages are one third to one tenth those in the US. Several approaches to this question will be presented including, the broadening of the typical engineering program to include specific focuses on leadership skills, the understanding of major issues such as the environment, population dynamics and poverty. A second approach will be getting our students involved in working on trans-national design projects that require working with other students over time and cultural boundaries so that they are better prepared to work in major corporations with internationally distributed engineering teams. A third project is to explore encouraging undergraduate involvement in teaching other undergraduates as a way of improving their depth of understanding of technical material and developing their communications and organizational skills. To add additional material to the program we will need to improve the efficiency with which our students learn new skills. This is likely to require an improved understanding by the faculty of the science of learning.
\end{abstract}

\section{Introduction}

The US faces some important public policy issues in deciding what skills we need to be teaching our young people if we are going to provide them with the opportunities to have the kind of life styles we believe are now possible. It is clear that science and technology now make it possible for many more people feed and house them selves in a comfortable manner than has ever been possible in the past. Scientific and technical information is being generated all around the world and we can expect both on the basis of population and the growth in the number of technically trained people worldwide that the fraction of it generated in the US is going to decrease. A public policy issue is how we take advantage of this growth in science and technology outside the US so as to improve our standard of living while helping the rest of the world to raise its. At the same time many well-trained people particularly in Asia will work for much less that we pay people in the US. The recent developments in communications and information technologies make it possible draw on this information as well as contribute to it. The question we have to answer is how do we train our young people to take advantage of these opportunities rather than being buried by the competition.

In the following I will attempt to make some suggestions of approaches that are practical enough to be implemented and different enough that it will take some new approaches the way we have been training our students. New approaches are not easy as we are all much more comfortable doing things we know how to do and there will be many people will give you lots reasons why none of these ideas will work.

\section{Leadership Training}


Leadership and supervisory rolls are one of the few ways our students can justify being paid more than their competition from parts of the world that pay much less. If we want our students to take leadership rolls we need think seriously about how we develop these skills in our students. This training can take many forms. One is technical skill. Another is organizational leadership. A third is the vision to anticipate important problems and define them in a way that they can be solved. Traditionally technical skills are the ones we spend most of our time on and this is the area where our students will have the most competition. Let us consider some approaches to this problem.

1. Let us consider first the possibility of requiring our undergraduates to play a significant roll in teaching other undergraduates. In teaching a class you learn the material better than when you took the course and you have to define the problems in a form the other students can learn to solve them. This will deepen their understanding of the technical material they are trying to teach. Students do not like to be embarrassed in front of their piers and they will prepare. They also get practice in organizing and presenting material. These are skills we are often told by industry that our students are lacking and skills they will need if they are lead organizations. It will also give the students taking the class more individual help than we can afford to give them with the current tight budgets and the demands on a professors time. Final as Carl Weiman pointed out to me from his experience with teaching assistants it changes the way the students study their own courses.

There are multiple ways to accomplish getting undergraduates involved in teaching. We have several programs where we are paying undergraduates as learning assistants in engineering, astronomy and physics at the University of Colorado and these seem to be effective for a large majority of the students involved. However resources limit the numbers of students. I would like to suggest that the educational value to the student doing the teaching is so large that we both give credit and require some teaching for graduation. This teaching could include helping run labs, running problem help sessions, grading papers and giving a lecture or two. Attending a seminar or two on learning theory, the physiology of the brain and problems in ethics with respect to grading, cheating and plagiarism could also be a requirement for the credit.

This semester I have had to be out of town several times and students in the class have given the lectures while I was gone. I have assigned two students to handle each lecture. For one lecture one of the students handed out 12 pages of lecture notes for the class he prepared and informal reports from other students on all of presentations have been favorable.

2. Many large corporations operate internationally with international design teams. It takes an understanding of multiple cultures as well as technical skills to lead these teams. One way to help our students develop the skills that will be needed to lead these teams is to have them work on such teams as students for design labs or homework problems. This can be done having courses taught jointly between the US and other schools. With web cams and the VOIP this can be done at relatively low cost. The hard part if finding two professors in different countries who want to work together and two administrations that will support their efforts. The ideal case is one where the professors both know each other and have complimentary areas of expertise that fit into a single course and the schedules match. Kurt 
Maute in our aerospace department has run a course with his colleague in Munich in advanced aircraft design. In this course there were three design teams one of which included students in both countries. Coordination problems and learning how to work together took some time and made the international team somewhat slower. However, the students were uniformly positive about the experience and the next time it is tried this coming fall some of the start up wrinkles with equipment, schedules etc. will be easier.

3. Another approach that has been widely discussed is giving our students a stronger background on the big picture. This means a basic understanding of issues like energy, clean water, the environment, and poverty and population dynamics. This also means understanding business and economics. Meeting regulatory requirements, environmental or safety standards or marketing requirements and time scales may be much harder than a technical design that works. This is difficult as there is already more technical material that we think our students need than we can get into the curriculum. Limiting the amount of technical material and finding ways to see that it is mastered is hard. To do this we need to improve the efficiency with which our students learn new material.

To do this we are going to have to take advantage of what is being learned about learning theory and the physiology of the brain. One place to start is the National Research Council report on How People Learn by the National Academy Press. Most of us have experience both in learning and teaching and we know some things that work and others that don't. Motivation is clearly important, however if we are going to improve our efficiency we also need to examine the way we present new material and how we couple it to what the students already know. Some things are best learned; hands on in the lab or on the job and others can be done with words or pictures. One approach that was suggested by a Russian colleague was a computer program that they had developed that builds up a profile of the students learning style as they work through the material. It looks at both the kind of wrong answers that are given to questions and how long the student takes to answer the question and they claimed to reduce the time required to learn new course material to $1 / 3$ of the standard approach. Unfortunately I have not been able to acquire a good description of the underling structure of this program however I think it is kind of target we need to shoot for if our students are acquire the breath of information we would like them to have.

\section{Taking Advantage of What is Being Learned Elsewhere and New Forms For the Distribution of Information}

Most of us have learned things in multiple ways ranging from the experience of building things that either worked or did not work to reading books, listening to lectures, watching TV and modeling things mathematically. The availability of material over the internet changes the cost and the ease of access to a very wide range a material to an extent that can dramatically change the way we do things and we need to seriously explore training our students on how to use it more effectively. Practically many times our students are more familiar with information resources that are available over the Internet than are their professors. However, they do not have the experience in filtering out good information from bad nor as extensive a frame of reference to sort out relevant information from an overwhelming volume of material. 
If they are to become leaders our students are going to need to know how to acquire information, organize it and act on the relevant parts of it. This information is being generated world wide and distributed in new formats. The research literature is now more extensive in almost any given field than any of us have time to read. For example in approximately the first year after the discovery of high temperature superconductors there were more than 4000 publications on the subject. Catalog information needed for engineering design such as electronic circuits is now so large and changes so rapidly that printed catalogs are often only a starting point and contain only a small fraction of the available information the design engineer could use. Most design engineers get much of the desired information from the web and various search engines become important and may lead to different data. The fact that data on the internet is available world wide at low to zero cost other than time means that those that know how to use these tools well will have a competitive advantage. Thus it is likely that we should be teaching courses that not only on information theory but also on how search engines like Google are designed. Students with systematic ways of acquiring and organizing information are going to have an advantage.

A second aspect of acquiring information for diverse sources worldwide is having enough understanding of the cultures where it is being generated to understand it and be able to use it. For example clinical trials in Eastern Europe have not used the same double blind standards used in the US and thus have to be evaluated differently. The differences in standards make it possible for them to explore therapies that we can not try within US rules but they also can lead to errors in interpretation that are less likely to occur here. There are also differences in the approach to problems in different cultures and in managing an international design team one does not want to assign a problem that requires getting ones hands dirty with a quick and dirty experimental solution to a theorist in country where getting ones hands dirty is looked down on as low class. The reverse is also true as there is nothing so practical as good theory that tells you exactly what to do and without a theory an experimentalist can do an endless number of experiments and still not understand what you need to do. If we are going to train leaders for worldwide engineering teams they are going to need to know how to capitalize on these different capabilities.

In closing it is my belief that the kind of difference in standard of living in the US and much of the rest of the world is unstable and at over the long haul it is in our interest to see that the standard of living in the rest of the world improves. It is going to take well-trained engineers in every part of the world to make this happen by solving the global problems like energy, poverty, global warming and clean water. 